\title{
QUANTITATIVE GEOMETRIC AND ARITHMETIC RESULTS ON PROJECTIVE SURFACES
}

\author{
HUNGZEN LIAO
}

(Communicated by Mei-Chi Shaw)

\begin{abstract}
In this paper, we improve Ru's defect relation and the height inequality in the case when $X$ is a normal projective surface and $D_{j}, 1 \leq j \leq q$, are big and asymptotic free divisors without irreducible common components on $X$. As a consequence, we derive a sharp result in the qualitative statement.
\end{abstract}

\section{INTRODUCTION AND STATEMENT OF THE MAIN RESUlts}

In recent years, there has been some significant progress in the study of qualitative and quantitative aspects of geometric and arithmetic properties of the complement of divisor in an algebraic projective variety. $\mathrm{Ru}$ (see [Ru15]) introduced the notion of Nevanlinna constant, denoted by $\operatorname{Nev}(D)$, for an effective Cartier divisor $D$ on a normal projective variety $X$. He then derived a new defect relation $\delta_{f}(D) \leq \operatorname{Nev}(D)$ for any Zariski dense holomorphic mapping $f: \mathbb{C} \rightarrow X$. As a consequence, he obtained the following result.

Theorem A ([Ru15, Theorem 5.6]). Let $X$ be a complex normal projective variety of dimension $\geq 2$, and $D_{1}, \ldots, D_{q}$ effective and big Cartier divisors in l-subgeneral position on $X$. Let $r_{i}>0$ be real numbers such that $D:=\sum_{i=1}^{q} r_{i} D_{i}$ is equidegree (such numbers exist due to Lemma 2.2). We further assume that the linear system $\left|N D_{i}\right|(i=1, \ldots, q)$ is base-point free for $N \geq N_{0}$. Let $f: \mathbb{C} \rightarrow X$ be a Zariski dense holomorphic map. Then, for $\epsilon_{0}>0$ small enough, which depends only on the given divisors,

$$
\sum_{j=1}^{q} r_{j} m_{f}\left(r, D_{j}\right)<\left(\frac{2 l \operatorname{dim} X}{q}-\epsilon_{0}\right)\left(\sum_{j=1}^{q} r_{j} T_{f, D_{j}}(r)\right) \|,
$$

where $\|$ means the inequality holds for all $r \in(0, \infty)$ except for a set of finite Lebesgue measure.

Recall that the divisors $D_{1}, \ldots, D_{q}$ on $X$ are said to be in l-subgeneral position on $X$ if for any subset of $l+1$ elements $\left\{i_{0}, \ldots, i_{l}\right\} \subset\{1, \ldots, q\}$,

$$
\operatorname{supp} D_{i_{0}} \cap \cdots \cap \operatorname{supp} D_{i_{l}}=\emptyset .
$$

When $l=\operatorname{dim} X$, then we say that the divisors $D_{1}, \ldots, D_{q}$ are in general position on $X$. The purpose of this paper is to improve Theorem A in the case when

Received by the editors November 30, 2015 and, in revised form, March 25, 2016; June 8, 2016; and July 12, 2016.

2010 Mathematics Subject Classification. Primary 32H30, 32H22, 11J97. 
$\operatorname{dim} X=2$ with an additional condition that the divisors $D_{1}, \ldots, D_{q}$ have no common irreducible components. The precise statement is as follows.

Main Theorem (Complex part). Let $X$ be a normal complex projective surface. Let $D_{1}, \ldots, D_{q}$ be effective, big Cartier divisors on $X$, and let the linear system $\left|N D_{i}\right|(i=1, \ldots, q)$ be base-point free for $N \geq N_{0}$. Assume that $D_{1}, \ldots, D_{q}$ have no irreducible components in common and are in l-subgeneral position. We further assume that $D:=\sum_{j=1}^{q} r_{j} D_{j}$ is equidegree for some positive real numbers $r_{j}$ (such $r_{j}$ always exist by Lemma 2.2). Let $f: \mathbb{C} \rightarrow X$ be holomorphic and Zariski dense. Then

$$
\sum_{j=1}^{q} r_{j} m_{f}\left(r, D_{j}\right) \leq \frac{4[(l+1) / 2]}{q(1+\alpha)}\left(\sum_{j=1}^{q} r_{j} T_{f, D_{j}}(r)\right) \|,
$$

where $\alpha=\frac{\min _{1 \leq j \leq q}\left(r_{j}^{2} D_{j}^{2}\right)}{384 q D^{2}},[x]$ denotes the greatest integer less than or equal to $x$, and $\|$ means the inequality holds for all $r \in(0, \infty)$ except for a set with finite Lebesgue measure.

Define the defect

$$
\delta_{f}(D):=\liminf _{r \rightarrow+\infty} \frac{m_{f}(r, D)}{T_{f, D}(r)} .
$$

Then, under the assumptions in the Main Theorem, we have the defect relation

$$
\delta_{f}(D) \leq \frac{4[(l+1) / 2]}{q(1+\alpha)} .
$$

Note that our techniques used in this paper to prove the above theorem are similar to Ru's method (see $\mathrm{Ru} 15]$ ). The main contribution of this paper is to use the joint filtrations lemma due to Corvaja and Zannier CZ04a (see Lemma 3.2 ) to lower the upper bound of the defect, under the additional assumption that $D_{1}, \ldots, D_{q}$ have no irreducible components in common. Furthermore, we give the explicit computation of the $\epsilon_{0}$ appearing in Theorem A. Note that our result also holds for any dimension of $X$. The reason we only focus on the case when $\operatorname{dim} X=2$ is the following sharp corollary of our Main Theorem, which is due to Levin [Lev09].

Corollary 1.1 ([Lev09, Theorem 11.5B]). Let $X$ be a nonsingular projective surface and $D_{1}, D_{2}, \ldots, D_{q}$ be effective and big divisors on $X$. Assume that $D_{1}, \ldots, D_{q}$ have no irreducible components in common and are in general position. If $q \geq 4$, then every holomorphic mapping $f: \mathbb{C} \rightarrow X \backslash \bigcup_{j=1}^{q} D_{j}$ must be algebraically degenerate.

Proof. According to Theorem 11.5 in [Lev09], we can reduce to the case when $\left|D_{i}\right|$ is base-point free (in particular, $D_{i}, 1 \leq i \leq q$, are nef) and $D_{i}$ is big for all $i$. If $f$ is not algebraically degenerate, then the above Main Theorem implies that $\delta_{f}(D)<1$, where $D=D_{1}+D_{2}+\cdots+D_{q}$. On the other hand, from the first main theorem, we have $\delta_{f}(D)=1$. This gives a contradiction. Therefore, $f$ must be algebraically degenerate.

On the arithmetic side, the counterpart of Theorem A in Diophantine approximation is stated as follows. For standard notation in Diophantine approximation, see Ru16].

Theorem B $\left(\left[\underline{\mathrm{Ru} 16}\right.\right.$, Theorem 4.1]). Let $k$ be a number field and $S \subset M_{k}$ be a finite set containing all archimedean places. Let $X$ be a normal projective variety with 
$\operatorname{dim} X \geq 2$ and let $D_{1}, \ldots, D_{q}$ be effective and big Cartier divisors in l-subgeneral position on $X$, both defined over $k$. Let $r_{i}>0$ be real numbers such that $D:=$ $\sum_{i=1}^{q} r_{i} D_{i}$ is equidegree (such numbers exist due to Lemma 2.2). We further assume that the linear system $\left|N D_{i}\right|(i=1, \ldots, q)$ is base-point free for $N \geq N_{0}$. Let $\lambda_{D_{j}, v}(x), 1 \leq j \leq q$, be the Weil function associated to $D_{j}$ for $v \in S$.

Then, for $\epsilon_{0}>0$ small enough,

$$
\sum_{j=1}^{q} \sum_{v \in S} r_{j} \lambda_{D_{j}, v}(x)<\left(\frac{2 l \operatorname{dim} X}{q}-\epsilon_{0}\right)\left(\sum_{j=1}^{q} r_{j} h_{D_{j}}(x)\right)
$$

holds for all $x \in X$ outside a Zariski closed subset $Z$ of $X$, where $h_{D_{j}}(x)=$ $\sum_{v \in M_{k}} \lambda_{D_{j}, v}(x)$.

Our theorem on the arithmetic side which improves Theorem B is as follows.

Main Theorem (Arithmetic Part). Let $k$ be a number field and $S \subset M_{k}$ a finite set containing all archimedean places. Let $X$ be a normal projective surface, and let $D_{1}, \ldots, D_{q}$ be effective and big Cartier divisors on $X$, both defined over $k$. Assume that the linear system $\left|N D_{i}\right|(i=1, \ldots, q)$ is base-point free for $N \geq N_{0}$. Assume that $D_{1}, \ldots, D_{q}$ have no irreducible components in common and are in l-subgeneral position. We further assume that $D:=\sum_{i=1}^{q} r_{j} D_{i}$ is equidegree for some positive real numbers $r_{j}$ (such $r_{j}$ always exist by Lemma 2.2). Let $\lambda_{D_{j}, v}(x), 1 \leq j \leq q$, be the Weil function associated to $D_{j}$ for $v \in S$. Then

$$
\sum_{j=1}^{q} \sum_{v \in S} r_{j} \lambda_{D_{j}, v}(x) \leq \frac{4[(l+1) / 2]}{q(1+\alpha)}\left(\sum_{j=1}^{q} r_{j} h_{D_{j}}(x)\right)
$$

holds for all $x \in X$ outside a Zariski closed subset $Z$ of $X$, where $\alpha=\frac{\min _{1 \leq j \leq q}\left(r_{j}^{2} D_{j}^{2}\right)}{384 q D^{2}}$, $[x]$ denotes the greatest integer less than or equal to $x$, and $h_{D_{j}}(x)=\sum_{v \in M_{k}} \lambda_{D_{j}, v}(x)$.

The above theorem gives the following corollary.

Corollary 1.2 ([Lev09, Theorem 11.5A]). Let $k$ be a number field and $S \subset M_{k}$ be a finite set containing all archimedean places. Let $X$ be a nonsingular projective surface and $D_{1}, \ldots, D_{q}$ effective and big Cartier divisors on $X$, both defined over a number field $k$. Assume that $D_{1}, \ldots, D_{q}$ have no irreducible components in common and are in general position. If $q \geq 4$, then any set of $(D, S)$-integral points of $X \backslash D$ is contained in a proper subvariety of $X$.

The proof of the result of the arithmetic case is similar to the complex case (see [Ru16]), so the rest of the paper will only focus on the complex part.

\section{NotATION AND PREPARATION LEMMAS}

We recall some notation. Let $X$ be a complex projective variety. For a Cartier divisor $D$ on $X$, the Weil function for $D$ is given by

$$
\lambda_{D}(x)=-\log \left\|s_{D}(x)\right\|,
$$

where $s_{D}$ is the canonical section of the line bundle $\mathcal{O}_{X}(D)$, i.e. $\left(s_{D}\right)=D$, and $\|\cdot\|$ is any continuous metric on $\mathcal{O}_{X}(D)$. It is well defined, up to a bounded term, independent of the choices of the metric. In the case when $X=\mathbb{P}^{n}(\mathbb{C})$ and 
$D=\{Q=0\} \subset \mathbb{P}^{n}(\mathbb{C})$ where $Q$ is a homogeneous polynomial of degree $d, \lambda_{D}$ can be chosen as, for $x=\left[x_{0}: \cdots: x_{n}\right] \in \mathbb{P}^{n}(\mathbb{C}) \backslash \operatorname{supp} D$,

$$
\lambda_{D}(x)=\log \frac{\left(\max _{0 \leq i \leq n}\left|x_{i}\right|^{d}\right) \cdot\|Q\|}{|Q(x)|},
$$

where $\|Q\|$ is the maximum of the norm of the coefficients of $Q$.

Let $f: \mathbb{C} \rightarrow X$ be a holomorphic map whose image is not contained in the support of $D$. The proximity function of $f$ with respect to $D$ is defined by

$$
m_{f}(r, D)=\int_{0}^{2 \pi} \lambda_{D}\left(f\left(r e^{i \theta}\right)\right) \frac{d \theta}{2 \pi} .
$$

The counting function of $f$ is defined by

$$
N_{f}(r, D)=\int_{1}^{r} \frac{n_{f}(t, D)}{t} d t
$$

where $n_{f}(t, D)$ is the number of zeros of $\rho \circ f$ inside $\{|z|<t\}$, counting multiplicities, and $\rho$ is a local defining function of $D$ (note that $n_{f}(t, D)$ is independent of the choice of $\rho$ ). We define the characteristic function by

$$
T_{f}(r, D):=m_{f}(r, D)+N_{f}(r, D) .
$$

The notions of the Weil function and the height function in the arithmetic (Diophantine approximation) side are defined in a similar way (see, for example, Lan87. or Vojcm $)$.

Next, we recall some definitions and lemmas from Levin's paper Lev09.

Definition 2.1 ([Lev09, Definition 9.6]). Suppose that $X$ is a projective variety of dimension $n$. Let $D=D_{1}+D_{2}+\cdots+D_{q}$ be a divisor on $X$ with $D_{i}$ effective. $D$ is said to have equidegree with respect to $D_{1}, D_{2}, \ldots, D_{q}$ if $D_{i} \cdot D^{n-1}=\frac{1}{q} D^{n}$ for $1 \leq i \leq q$.

Lemma 2.2 ([Lev09, Lemma 9.7]). Let $X$ be a projective variety of dimension $n$. If $D_{j}, 1 \leq j \leq q$, are big and nef, then there exist positive real numbers $r_{j}$ such that $D=\sum_{j=1}^{q} r_{j} D_{j}$ is of equidegree with respect to $r_{1} D_{1}, \ldots, r_{q} D_{q}$.

Proof. We follow the simple proof given by Autissier [Aut1]. Let

$$
\triangle:=\left\{\left(t_{1}, \ldots, t_{q}\right) \in \mathbb{R}_{+}^{q} \mid t_{1}+\cdots+t_{q}=1\right\} .
$$

Define a map $g: \triangle \rightarrow \triangle$ by letting, for $t=\left(t_{1}, \ldots, t_{q}\right) \in \triangle$,

$$
g(t)=\left(\frac{\phi(t)}{\left(\sum_{j=1}^{q} t_{j} D_{j}\right)^{n-1} \cdot D_{1}}, \cdots, \frac{\phi(t)}{\left(\sum_{j=1}^{q} t_{j} D_{j}\right)^{n-1} \cdot D_{q}}\right),
$$

where $\phi(t):=\left(\sum_{i=1}^{q} \frac{1}{\left(\sum_{j=1}^{q} t_{j} D_{j}\right)^{n-1} \cdot D_{i}}\right)^{-1}$. By Brouwer's fixed point theorem, there exists a point $x=\left(r_{1}, \ldots, r_{q}\right)$ such that $g(x)=x$, i.e. $\phi(x)=\left(\sum_{j=1}^{q} r_{j} D_{j}\right)^{n-1}$. $\left(r_{i} D_{i}\right)$ for $i=1, \ldots, q$. This implies, by summing all $i$, that $q \phi(x)=\left(\sum_{j=1}^{q} r_{j} D_{j}\right)^{n}$. Thus

$$
\frac{1}{q}\left(\sum_{j=1}^{q} r_{j} D_{j}\right)^{n}=\phi(x)=\left(r_{i} D_{i}\right) \cdot\left(\sum_{j=1}^{q} r_{j} D_{j}\right)^{n-1}
$$

which proves the lemma. 
Lemma 2.2 tells us that we can always make the given big and nef divisors to be of equidegree without changing their supports since the divisors $r_{j} D_{j}$ and $D_{j}$ are of the same supports. This means the notion of equidegree, rather than the condition of linear equivalence for the divisors $D=D_{1}+\cdots+D_{q}$, would be a correct (or proper) notion in the study of degeneracy of holomorphic mappings $f: \mathbb{C} \rightarrow X \backslash D$. In the surface case, we denote by $\left(D_{i} . D_{j}\right)$ the intersection number of $D_{i}$ and $D_{j}$. We also denote $(D . D)$ by $D^{2}$.

We also need the following results in algebraic geometry. Let $X$ be a complex projective algebraic manifold of dimension two. Let $D$ be a divisor on $X$. Let $\mathcal{O}(D)$ be the invertible sheaf associated to the divisor $D$ on $X$. For $i=0,1,2$, let $h^{i}(D)=\operatorname{dim} H^{i}(X, \mathcal{O}(D))$ (we sometimes also write $H^{i}(X, \mathcal{O}(D))$ as $H^{i}(X, D)$ for simplicity).

Lemma 2.3 (Laz04, Corollary 1.4.41]). Suppose that $D$ is a nef divisor on a projective surface on $X$. Then

$$
h^{0}(N D)=\frac{D^{2} N^{2}}{2}+O(N) .
$$

In particular, $D^{2}$ is positive if and only if $D$ is big.

Lemma 2.4 (Aut1, Lemma 4.2]). Let $X$ be a projective surface. Let $F$ be a big and base-point free Cartier divisor and $D$ a Cartier divisor such that $D-F$ is also nef. Let $\beta>0$ be a positive real number. Then for any positive integer $N, k$ with $1 \leq k \leq \beta N$, we have

$$
h^{0}(N D-k F) \geq \frac{D^{2} N^{2}}{2}-(D . F) N k+\frac{F^{2}}{2} \min \left\{k^{2}, N^{2}\right\}+O(N),
$$

where $O(N)$ depends on $\beta$.

Lemma 2.5. Let $D$ and $F$ be the same as above on a projective surface $X$. We further assume that $\frac{D^{2}}{(D . F)} \geq 1$. Then

$$
\sum_{k=1}^{\infty} h^{0}(N D-k F) \geq\left(\frac{D^{2}}{4(D \cdot F)}+\frac{F^{2}}{24 D^{2}}\right) N h^{0}(N D)+O\left(N^{2}\right) .
$$

Proof. Using Lemma 2.4 with $\beta=\frac{D^{2}}{2(D . F)} \geq \frac{1}{2}$, we get, by noticing that $\min \left\{k^{2}, N^{2}\right\} \geq$ $\min \left\{k^{2}, N^{2} / 4\right\}$,

$$
\begin{aligned}
& \sum_{k=1}^{\infty} h^{0}(N D-k F) \\
\geq & \sum_{k=1}^{[\beta N]}\left(\frac{D^{2} N^{2}}{2}-(D \cdot F) N k+\frac{F^{2}}{2} \min \left\{k^{2}, \frac{N^{2}}{4}\right\}\right)+O\left(N^{2}\right) \\
\geq & \frac{\left(D^{2}\right)^{2} N^{3}}{4(D \cdot F)}-(D \cdot F) \frac{\left(D^{2}\right)^{2} N^{3}}{8(D \cdot F)^{2}}+\sum_{k=1}^{[N / 2]-1} \frac{F^{2}}{2} k^{2}+\sum_{[N / 2]}^{[\beta N]} \frac{F^{2}}{8} N^{2}+O\left(N^{2}\right) \\
= & \frac{\left(D^{2}\right)^{2}}{8(D \cdot F)} N^{3}+\frac{F^{2}}{48} N^{3}+\frac{F^{2}}{8} \beta N^{3}-\frac{F^{2}}{16} N^{3}+O\left(N^{2}\right) \\
= & \left(\frac{D^{2}}{4(D \cdot F)}+\frac{F^{2}}{D^{2}}\left(\frac{\beta}{4}-\frac{1}{12}\right)\right) \frac{D^{2}}{2} N^{3}+O\left(N^{2}\right)
\end{aligned}
$$




$$
\begin{aligned}
& \geq\left(\frac{D^{2}}{4(D \cdot F)}+\frac{F^{2}}{24 D^{2}}\right) \frac{D^{2}}{2} N^{3}+O\left(N^{2}\right) \\
& =\left(\frac{D^{2}}{4(D \cdot F)}+\frac{F^{2}}{24 D^{2}}\right) N h^{0}(N D)+O\left(N^{2}\right) .
\end{aligned}
$$

\section{Proof of the Main Theorem}

The proof of the Main Theorem relies on the notion of Nevanlinna constant, $\operatorname{Nev}(D)$, introduced by $\mathrm{Ru}$ (see $\mathrm{Ru} 15]$ ), and his results in terms of $\operatorname{Nev}(D)$. Let $X$ be a normal projective variety and $D$ an effective Cartier divisor on $X$. Note that the condition of normality of $X$ is assumed so that $\operatorname{ord}_{E} D$ (called the coefficient of $D$ in $E$ ) is defined for any prime divisor $E$ and any effective Cartier divisor $D$ on $X$ (see Laz04, Remark 1.1.4]). For any section $s \in H^{0}(X, D)$, we use $\operatorname{ord}_{E} s$ or $\operatorname{ord}_{E}(s)$ to denote the coefficients of $(s)$ in $E$, where $(s)$ is the divisor on $X$ associated to $s$.

Definition 3.1. Let $X$ be a normal complex projective variety and $D$ an effective Cartier divisor on $X$. The Nevanlinna constant of $D$, denoted by $\operatorname{Nev}(D)$, is given by

$$
\operatorname{Nev}(D):=\inf _{N}\left(\inf _{\left\{\mu_{N}, V_{N}\right\}} \frac{\operatorname{dim} V_{N}}{\mu_{N}}\right)
$$

where the infimum "inf" is taken over all positive integers $N$ and the infimum " $\inf _{\left\{\mu_{N}, V_{N}\right\}} "$ is taken over all pairs $\left\{\mu_{N}, V_{N}\right\}$, where $\mu_{N}$ is a positive real number and $V_{N} \subseteq H^{0}(X, N D)$ is a linear subspace with $\operatorname{dim} V_{N} \geq 2$ such that, for all $P \in \operatorname{supp} D$, there exists a basis $B$ of $V_{N}$ with

$$
\sum_{s \in B} \operatorname{ord}_{E}(s) \geq \mu_{N} \operatorname{ord}_{E}(N D)
$$

for all irreducible components $E$ of $D$ passing through $P$. If $h^{0}(N D) \leq 1$ for all positive integers $N$, we define $\operatorname{Nev}(D)=+\infty$.

Theorem C ([ $\mathrm{Ru15}$, Main Theorem]). Let $X$ be a complex normal projective variety and $D$ an effective Cartier divisor on $X$. Then, for every $\epsilon>0$,

$$
m_{f}(r, D) \leq(\operatorname{Nev}(D)+\epsilon) T_{f}(r, D) \|
$$

holds for any Zariski dense holomorphic mapping $f: \mathbb{C} \rightarrow X$.

The following joint filtrations lemma is crucial to the proof of our Main Theorem.

Lemma 3.2 ([CZ04a, Lemma 3.2]). Let $V$ be a vector space of finite dimension $d$ over a field $k$. Let $V=W_{1} \supset W_{2} \supset W_{3} \supset \ldots \supset W_{h}$ and $V=W_{1}^{*} \supset W_{2}^{*} \supset W_{3}^{*} \supset$ $\ldots \supset W_{h^{*}}^{*}$ be two filtrations on $V$. Then there exists a basis of $V$ that contains a basis of each $W_{j}$ and $W_{j}^{*}$.

Proof of the Main Theorem (complex part). The proof uses Theorem C, so we need to compute the Nevanlinna constant. By taking $N \geq N_{0}$, we can assume that $D_{j}, 1 \leq j \leq q$, are base-point free. By the assumption that $D$ has equidegree with respect to $r_{1} D_{1}, \ldots, r_{q} D_{q}$, we have, with $D:=\sum_{j=1}^{q} r_{j} D_{j}$,

$$
\left(r_{i} D_{i} \cdot D\right)=\frac{1}{q} D^{2}
$$


To simplify the notation, we write

$$
\alpha:=\frac{\min _{1 \leq j \leq q}\left(r_{j}^{2} D_{j}^{2}\right)}{384 q D^{2}} .
$$

Choose positive rational numbers $a_{j}, 1 \leq j \leq q$, such that

$$
\left|a_{j}-r_{j}\right| \leq \frac{\delta_{1}\left(\min _{1 \leq j \leq q} r_{j}\right)}{2} \min \left\{1, \frac{q(1+2 \alpha)}{4[(l+1) / 2]}\right\}
$$

and, for $i=1, \ldots, q$,

$$
\left|\frac{\left(a_{i} D_{i} \cdot D^{\prime}\right)}{D^{\prime 2}}-\frac{1}{q}\right|<\delta_{2}
$$

where $D^{\prime}:=\sum_{j=1}^{q} a_{j} D_{j}$ and $\delta_{1}, \delta_{2}$ will be chosen below (see (17) and (13)). Note that with our choice of $\delta_{1}$ and $\left|a_{j}-r_{j}\right| \leq \frac{r_{j}}{2}$, we have

$$
D^{\prime 2} \geq \frac{1}{4} D^{2} \text { and } D^{2} \geq \frac{1}{4} D^{\prime 2}
$$

Now, for $P \in \operatorname{supp} D$, let $D_{P}^{\prime}:=\sum_{i: P \in \operatorname{supp} D_{i}} a_{i} D_{i}$. Since $D_{1}, \ldots, D_{q}$ are in $l$ subgeneral position and any two of $D_{1}, \ldots, D_{q}$ have no common components, we can write

$$
D_{P}^{\prime}:=D_{P, 1}^{\prime}+D_{P, 2}^{\prime},
$$

where $D_{P, 1}^{\prime}$ and $D_{P, 2}^{\prime}$ are each a sum of no more than $[(l+1) / 2]$ terms of the $a_{i} D_{i}$, and $D_{P, 1}^{\prime}$ and $D_{P, 2}^{\prime}$ have no irreducible components in common. Let $d$ be the product of the denominators of $a_{1}, \ldots, a_{q}$ and consider $V_{N}:=H^{0}\left(X, N d D^{\prime}\right)$. Note that $D^{\prime}$ is only a $\mathbb{Q}$-divisor, so we need to multiply $d$ by $D^{\prime}$ to make $d D^{\prime}$ be an (integral) divisor. We consider the following two filtrations of $V_{N}$ :

$$
W_{m}:=H^{0}\left(X, N d D^{\prime}-m d D_{P, 1}^{\prime}\right), W_{m}^{*}:=H^{0}\left(X, N d D^{\prime}-m d D_{P, 2}^{\prime}\right), m=0,1, \ldots
$$

Using the filtration lemma above, we obtain a basis $B$ that contains a basis for each $W_{m}$ and $W_{m}^{*}$. Let $E$ be an irreducible component of $D$ which contains $P$. Then $E$ is contained either in $d D_{P, 1}^{\prime}$ or $d D_{P, 2}^{\prime}$, but not both. Without loss of generality, we assume that $E$ is an irreducible component of $d D_{P, 1}^{\prime}$, and thus $\operatorname{ord}_{E}\left(d D^{\prime}\right)=$ $\operatorname{ord}_{E}\left(d D_{P, 1}^{\prime}\right)$. We also note that $\operatorname{ord}_{E} s \geq m \operatorname{ord}_{E}\left(d D_{P, 1}^{\prime}\right)$ for any $s \in H^{0}\left(X, N d D^{\prime}-\right.$ $\left.m d D_{P, 1}^{\prime}\right)$ (regarded as a subspace of $H^{0}\left(X, N d D^{\prime}\right)$ ). Hence

$$
\begin{aligned}
& \frac{1}{\operatorname{ord}_{E}\left(N d D^{\prime}\right)} \sum_{s \in B} \operatorname{ord}_{E} s=\frac{1}{\operatorname{ord}_{E}\left(N d D_{P, 1}^{\prime}\right)} \sum_{s \in B} \operatorname{ord}_{E} s \\
\geq & \frac{1}{N} \sum_{m=0}^{\infty} m\left(h^{0}\left(N d D^{\prime}-m d D_{P, 1}^{\prime}\right)-h^{0}\left(N d D^{\prime}-(m+1) d D_{P, 1}^{\prime}\right)\right. \\
= & \frac{1}{N} \sum_{k=1}^{\infty} h^{0}\left(N d D^{\prime}-k d D_{P, 1}^{\prime}\right) .
\end{aligned}
$$


Noticing that $\frac{D^{\prime 2}}{\left(D^{\prime} \cdot D_{P, 1}^{\prime}\right)} \geq 1$, by Lemma 2.5 , we get

$$
\begin{aligned}
& \sum_{k=1}^{\infty} h^{0}\left(N d D^{\prime}-k d D_{P, 1}^{\prime}\right) \\
\geq & \left(\frac{\left(d D^{\prime}\right)^{2}}{4\left(d D^{\prime} \cdot d D_{P, 1}^{\prime}\right)}+\frac{\left(d D_{P, 1}^{\prime}\right)^{2}}{24\left(d D^{\prime}\right)^{2}}\right) N h^{0}\left(N d D^{\prime}\right)+O\left(N^{2}\right) \\
= & \left(\frac{D^{\prime 2}}{4\left(D^{\prime} \cdot D_{P, 1}^{\prime}\right)}+\frac{D_{P, 1}^{\prime 2}}{24 D^{\prime 2}}\right) N h^{0}\left(N d D^{\prime}\right)+O\left(N^{2}\right) .
\end{aligned}
$$

Therefore,

$$
\frac{1}{\operatorname{ord}_{E}\left(N d D^{\prime}\right)} \sum_{s \in B} \operatorname{ord}_{E} s \geq\left(\frac{D^{\prime 2}}{4\left(D^{\prime} \cdot D_{P, 1}^{\prime}\right)}+\frac{D_{P, 1}^{\prime 2}}{24 D^{\prime 2}}\right) h^{0}\left(N d D^{\prime}\right)+O(N) .
$$

We now estimate each term appearing above. Since $D_{P, 1}^{\prime}$ is a sum of no more than $[(l+1) / 2]$ terms of the $a_{i} D_{i}$, by using (8),

$$
\frac{\left(D^{\prime} \cdot D_{P, 1}^{\prime}\right)}{D^{\prime 2}} \leq \frac{[(l+1) / 2] \max _{i, P \in D_{i}}\left\{\left(D^{\prime} \cdot\left(a_{i} D_{i}\right)\right)\right\}}{D^{\prime 2}} \leq[(l+1) / 2]\left(\frac{1}{q}+\delta_{2}\right) .
$$

Also notice that, using (6), (7) and (9), we get

$$
\begin{aligned}
\left(D_{P, 1}^{\prime} \cdot D_{P, 1}^{\prime}\right) & \geq \min _{1 \leq i \leq q}\left(a_{i} D_{i} \cdot a_{i} D_{i}\right) \geq \frac{1}{4} \min _{1 \leq i \leq q}\left(r_{i} D_{i} \cdot r_{i} D_{i}\right) \\
& =96 q(D \cdot D) \alpha \geq 24 q\left(D^{\prime} \cdot D^{\prime}\right) \alpha .
\end{aligned}
$$

Combining (10), (11), and (12), we get

$$
\begin{aligned}
& \frac{1}{\operatorname{ord}_{E}\left(N d D^{\prime}\right)} \sum_{s \in B} \operatorname{ord}_{E} s \\
\geq & \left(\frac{q}{4[(l+1) / 2]\left(1+q \delta_{2}\right)}+q \alpha\right) \frac{d^{2} D^{\prime 2}}{2} N^{2}+O(N) \\
\geq & \frac{q}{4[(l+1) / 2]}\left(\frac{1}{1+q \delta_{2}}+4 \alpha\right) h^{0}\left(N d D^{\prime}\right)+O(N) \\
= & \frac{q(1+3 \alpha)}{4[(l+1) / 2]} h^{0}\left(N d D^{\prime}\right)+O(N),
\end{aligned}
$$

where the last equality holds when choosing

$$
\delta_{2}=\frac{\alpha}{q(1-\alpha)} .
$$

Hence, from the definition of Nevanlinna's constant (see (5)), we obtain

$$
\operatorname{Nev}\left(d D^{\prime}\right) \leq \frac{4[(l+1) / 2]}{q(1+3 \alpha)}
$$

Applying Theorem $\mathrm{C}$ with $\epsilon=\frac{4[(l+1) / 2] \alpha}{q(1+3 \alpha)(1+2 \alpha)}$, we get

$$
\sum_{j=1}^{q} a_{j} d m_{f}\left(r, D_{j}\right) \leq\left(\frac{4[(l+1) / 2]}{q(1+2 \alpha)}\right)\left(\sum_{j=1}^{q} a_{j} d T_{f}\left(r, D_{j}\right)\right) \| .
$$


By canceling $d$ on both sides, we have

$$
\sum_{j=1}^{q} a_{j} m_{f}\left(r, D_{j}\right) \leq\left(\frac{4[(l+1) / 2]}{q(1+2 \alpha)}\right)\left(\sum_{j=1}^{q} a_{j} T_{f}\left(r, D_{j}\right)\right) \| .
$$

We now use this result to derive our desired result. Notice that (7) gives us

$$
\sum_{j=1}^{q} r_{j} m_{f}\left(r, D_{j}\right) \leq \sum_{j=1}^{q} a_{j} m_{f}\left(r, D_{j}\right)+\frac{\delta_{1}}{2}\left(\min _{1 \leq j \leq q} r_{j}\right) \sum_{j=1}^{q} m_{f}\left(r, D_{j}\right)
$$

and

(16) $\sum_{j=1}^{q} a_{j} T_{f, D_{j}}(r) \leq \sum_{j=1}^{q} r_{j} T_{f, D_{j}}(r)+\frac{\delta_{1}}{2}\left(\min _{1 \leq j \leq q} r_{j}\right) \frac{q(1+2 \alpha)}{4[(l+1) / 2]} \sum_{j=1}^{q} T_{f, D_{j}}(r)$.

Using (14), (15), (16), together with the first main theorem, we get

$$
\begin{aligned}
& \sum_{j=1}^{q} r_{j} m_{f}\left(r, D_{j}\right) \\
\leq & \frac{4[(l+1) / 2]}{q(1+2 \alpha)}\left(\sum_{j=1}^{q} a_{j} T_{f}\left(r, D_{j}\right)\right)+\frac{\left(\min r_{j}\right) \delta_{1}}{2}\left(\sum_{j=1}^{q} T_{f}\left(r, D_{j}\right)\right) \| \\
\leq & \frac{4[(l+1) / 2]}{q(1+2 \alpha)}\left(\sum_{j=1}^{q} r_{j} T_{f}\left(r, D_{j}\right)+\frac{\left(\min r_{j}\right) \delta_{1}}{2} \frac{q(1+2 \alpha)}{4[(l+1) / 2]} \sum_{j=1}^{q} T_{f}\left(r, D_{j}\right)\right) \\
& +\frac{\delta_{1}}{2} \sum_{j=1}^{q} r_{j} T_{f}\left(r, D_{j}\right) \| \\
\leq & \left(\frac{4[(l+1) / 2]}{q(1+2 \alpha)}+\delta_{1}\right) \sum_{j=1}^{q} r_{j} T_{f}\left(r, D_{j}\right) \| \\
\leq & \left(\frac{4[(l+1) / 2]}{q(1+\alpha)}\right) \sum_{j=1}^{q} r_{j} T_{f}\left(r, D_{j}\right) \|,
\end{aligned}
$$

with

$$
\delta_{1}=\min \left\{1, \frac{4[(l+1) / 2]}{q} \frac{\alpha}{(1+\alpha)(1+2 \alpha)}\right\} .
$$

This finishes the proof of the Main Theorem.

\section{ACKNOWLEDGMEnts}

The author thanks his advisor, Professor Min Ru, for introducing this fantastic field and many discussions. He also wants to thank the referee for the careful reading and many helpful suggestions.

\section{REFERENCES}

[Aut1] Pascal Autissier, Géométries, points entiers et courbes entières (French, with English and French summaries), Ann. Sci. Éc. Norm. Supér. (4) 42 (2009), no. 2, 221-239. MR 2518077

[Car] H. Cartan, Sur les zéros des combinaisions linéarires de p fonctions holomorpes données, Mathematica (Cluj) 7 (1933), 5-29. 
[CZ04a] P. Corvaja and U. Zannier, On integral points on surfaces, Ann. of Math. (2) 160 (2004), no. 2, 705-726, DOI 10.4007/annals.2004.160.705. MR2123936

[CZ04a] Pietro Corvaja and Umberto Zannier, On a general Thue's equation, Amer. J. Math. 126 (2004), no. 5, 1033-1055. MR.2089081

[EF02] Jan-Hendrik Evertse and Roberto G. Ferretti, Diophantine inequalities on projective varieties, Int. Math. Res. Not. 25 (2002), 1295-1330, DOI 10.1155/S107379280210804X. MR 1903776

[EF08] Jan-Hendrik Evertse and Roberto G. Ferretti, A generalization of the Subspace Theorem with polynomials of higher degree, Diophantine approximation, Dev. Math., vol. 16, SpringerWienNewYork, Vienna, 2008, pp. 175-198, DOI 10.1007/978-3-211-74280-8_9. MR2487693

[Ful98] William Fulton, Intersection theory, 2nd ed., Ergebnisse der Mathematik und ihrer Grenzgebiete. 3. Folge. A Series of Modern Surveys in Mathematics [Results in Mathematics and Related Areas. 3rd Series. A Series of Modern Surveys in Mathematics], vol. 2, Springer-Verlag, Berlin, 1998. MR1644323

[Lan87] Serge Lang, Fundamentals of Diophantine geometry, Springer-Verlag, New York, 1983. MR715605

[Laz04] Robert Lazarsfeld, Positivity in algebraic geometry. I, Classical setting: line bundles and linear series, Ergebnisse der Mathematik und ihrer Grenzgebiete. 3. Folge. A Series of Modern Surveys in Mathematics [Results in Mathematics and Related Areas. 3rd Series. A Series of Modern Surveys in Mathematics], vol. 48, Springer-Verlag, Berlin, 2004. MR2095471

[Lev09] Aaron Levin, Generalizations of Siegel's and Picard's theorems, Ann. of Math. (2) 170 (2009), no. 2, 609-655, DOI 10.4007/annals.2009.170.609. MR2552103

[Lev14] Aaron Levin, On the Schmidt subspace theorem for algebraic points, Duke Math. J. 163 (2014), no. 15, 2841-2885, DOI 10.1215/00127094-2827017. MR 3285859

[LR14] Hungzen Liao and Min Ru, A note on the Second Main Theorem for holomorphic curves into algebraic varieties, Bull. Inst. Math. Acad. Sin. (N.S.) 9 (2014), no. 4, 671-684. MR3309947

[Ru97] Min Ru, On a general form of the second main theorem, Trans. Amer. Math. Soc. 349 (1997), no. 12, 5093-5105, DOI 10.1090/S0002-9947-97-01913-2. MR1407711

[Ru04] Min Ru, A defect relation for holomorphic curves intersecting hypersurfaces, Amer. J. Math. 126 (2004), no. 1, 215-226. MR2033568

[Ru09] Min Ru, Holomorphic curves into algebraic varieties, Ann. of Math. (2) 169 (2009), no. 1, 255-267, DOI 10.4007/annals.2009.169.255. MR2480605

[Ru15] M. Ru, A defect relation for holomorphic curves intersecting general divisors in projective varieties, J. of Geometric Analysis, first online: 15 October 2015.

[Ru16] M. Ru, A general Diophantine inequality, Functiones et Approximatio, to appear.

[Voj87] Paul Vojta, Diophantine approximations and value distribution theory, Lecture Notes in Mathematics, vol. 1239, Springer-Verlag, Berlin, 1987. MR883451

[Voj97] Paul Vojta, On Cartan's theorem and Cartan's conjecture, Amer. J. Math. 119 (1997), no. 1, 1-17. MR 1428056

[Vojcm] P. Vojta, Diophantine approximation and Nevanlinna theory, CIME notes, page 231, 2007.

Department of Mathematics, University of Houston, 4800 Calhoun Road, Houston, TEXAs 77004

E-mail address: lhungzen@math.uh.edu 